\title{
AlO-Herbstkongress 2015: Interessante Daten aus der klinischen Krebsforschung
}

\author{
Im Rahmen des Herbstkongresses der Arbeitsgemeinschaft \\ Internistische Onkologie (AIO) in Berlin stellten die Arbeits- \\ und Studiengruppen der AIO ihre aktuellen onkologischen \\ Studien vor. Das ONKO-Internetportal informiert in Exper- \\ teninterviews über die wichtigsten Themen und Daten vom \\ Kongress.
}

\section{Immuntherapie gewinnt in vielen Indikationen an Bedeutung}

Prof. Arndt Vogel, Hannover, erläutert den Stand der klinischen Forschung bei der Therapie der Hepatobiliären Tumoren und geht dabei auf vielversprechende Daten mit Checkpoint-Inhibitoren beim hepatozellulären Karzinom ein.

Auch für Nierenzellkarzinome, Kopf-Hals-Tumoren und Weichteilsarkome gewinnt die Immuntherapie an Bedeutung, wie Prof. Viktor Grünwald, Hannover, im Interview mit dem ONKO-Internetportal erklärt. So wurden auf dem AIO-Kongress Daten aus Phase-III-Studien zur Immuntherapie präsentiert, aber auch neue Ansätze zum Therapiemanagement und zur Verbesserung der Lebensqualität diskutiert.

Für die metastasierten Formen des Kolorektalkarzinoms besteht aus der Sicht von Prof. Arnold die aktuelle Herausforderung darin, therapierelevante Biomarker zu finden. Im Rahmen der AIO werde hier Pionierarbeit geleistet, betont Arnold.

Beim Expertengespräch des ONKO-Internetportals zum Pankreaskarzinom diskutierten Fachleute, nach welchen Parametern sich der Einsatz neuer Substanzen richtet und welche Patientengruppen von den Therapiekonzepten besonders profitieren könnten.

\section{Viele neue Therapieoptionen beim Lungenkarzinom}

Dr. Martin Sebastian, Sprecher der AIO-Arbeitsgruppe Thorakale Onkologie, hebt im Interview die „Welle“ neuer Therapieoptionen beim Lungenkarzinom hervor. Exemplarisch stellt er einige wichtige Studienkonzepte vor.

Die neuen Therapiekonzepte haben auch ihren Niederschlag in der aktualisierten Onkopedia-Leitlinie zum Lungenkrebs gefunden, wie Dr. Wilfried Eberhardt, Essen, im Interview erläutert. Für Eberhardt zeichnet sich diese Leitlinie gegenüber der S3-Leitlinie des Leitlinienprogramms Onkologie u. a. durch ihre jährliche Aktualisierung aus.
Prof. Frank Griesinger, Oldenburg, berichtet live vom Kongress über den neuesten Stand der CRISP-Studie, die die AIO gemeinsam mit anderen Fachgesellschaften durchführt. In diese prospektive Registerstudie sollen in den nächsten drei Jahren über 8000 Patienten mit nichtkleinzelligem Lungenkarzinom eingeschlossen werden. Sie soll als Plattform für verschiedene Studienmodule dienen, so Griesinger.

\section{Erfolgreiches Mentoring für junge Onkologen}

Welche Erfolge das AIO-Mentoringprogramm „Young Medical Oncologists" für junge Onkologen aufweisen kann, zeigt der Start der MATEO-Studie beim fortgeschrittenen Magenkarzinom - so Prof. Florian Lordick, Leipzig und Dr. Georg Martin Haag, Heidelberg, im Interview. Die Studie untersucht den Stellenwert einer Erhaltungstherapie mit S-1 nach dreimonatiger Kombinationschemotherapie in der Erstlinientherapie des metastasierten Magenkarzinoms.

Prof. Ralf-Dieter Hofheinz, Mannheim, Vorsitzender der AIO Arbeitsgruppe Lebensqualität und Patient Reported Outcomes, berichtet über die wachsende Bedeutung der Erfassung der Lebensqualität und die Notwendigkeit neue Fragebögen zu entwickeln. Darüber hinaus betont er, dass in Zulassungsstudien das Thema Lebensqualität zunehmend wichtiger wird.

Einen generellen Einblick in die Arbeit der AIO-StudiengGmbH gibt Dr. Aysun Karatas, Geschäftsführerin der AIOStudien-gGmbH. Die 2007 innerhalb der AIO gegründete Organisation unterstütze AIO-Mitglieder bei der Planung und Durchführung von europaweiten onkologischen Studien und Registerstudien, so Karatas.

Alle Interviews können online aufgerufen unter www.krebsgesellschaft.de/kongresse.html oder unter diesem QR-Code:

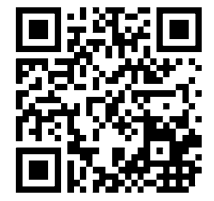

